\title{
La perspectiva post-cualitativa en la investigación educativa: genealogía, movimientos, posibilidades y tensiones
}

The post-qualitative approach in educational research: genealogy, strands, possibilities and tensions

\author{
Fernando Hernández-Hernández*1 \\ fdohernandez@ub.edu \\ Beatriz Revelles Benavente** \\ beatrizrevelles@ugr.es \\ * Universidad de Barcelona, España \\ **Universidad de Granada, España
}

\begin{abstract}
Resumen:
En este artículo se trata de situar una cierta genealogía de algunos de los movimientos que se vinculan en torno a la denominada perspectiva post-cualitativa en investigación. Estos movimientos han ido surgiendo como respuesta a los intentos, desde la década de los años 90 del pasado siglo, de positivizar, disciplinar y objetivar la investigación cualitativa bajo estándares y rúbricas que fijan los caminos a seguir tanto en la formación de investigadores como en la conceptualización y la práctica de la investigación. Esta tendencia, en el caso de Estados Unidos, que es donde está adquiriendo una mayor relevancia, queda reafirmada cuando en 2002 el National Research Council publica un documento en el que se establece cómo ha de ser la investigación científica en educación: replicable, generalizable, empírica y prefe-
\end{abstract}

\begin{abstract}
:
This article aims to establish the genealogy of the strands that are linked to the so-called post-qualitative approach in research. These strands have emerged as a response to the attempts, since the 1990s, to positivize and objectify qualitative research by applying to it standards and rubrics which seek to define researcher training and the conceptualization and practice of research. This tendency, in the case of the United States where it is acquiring great relevance, is reaffirmed in 2002 when the National Research Council publishes a document that establishes how scientific research in education should be: replicable, generalizable, empirical and preferably experimental. This decision is part of a neoliberal form of governance that seeks to establish a unified vision of what reality should be and the role that research
\end{abstract}

1 Dirección para correspondencia (correspondence address):

Fernando Hernández-Hernández. Universidad de Barcelona. Departamento de Artes Visuales y Diseño. Unidad de Pedagogías Culturales. Facultad de Bellas Artes. Pau Gargallo, 4. 08028 Barcelona (España). 
La perspectiva post-cualitativa en la investigación educativa: genealogía, movimientos, posibilidades y tensiones

Fernando Hernández-Hernández y Beatriz Revelles Benavente

rentemente experimental. Decisión que forma parte de una gobernabilidad neoliberal que pretende establecer una visión unificada respecto a lo que ha de ser la realidad y el papel que la investigación ha de jugar en este propósito. Este movimiento, que se nutre de diferentes referentes y que se proyecta en varias direcciones cuestiona con argumentos la fundamentación onto-espistemológica-metodológica y ética que rige este intento normativizador, al tiempo que rehúye la explicitación de modos de hacer investigación que predetermine los sentidos abiertos e imprevisibles de todo proceso de indagación y plantea otras maneras de conceptualizar lo que un proceso de investigación puede llegar a ser.

\section{Palabras clave:}

Post-cualitativa; indagación; post-humanismo; nuevo materialismo; rizoma; nueva ontología. should have in this reality. The post-qualitative approach, which feeds on different referents and projects itself in various directions, questions the onto-espistemological-methodological and ethical foundations that drive this normative attempt, at the same time as it avoids ways of doing research that predetermine the open and unpredictable meanings to which processes of inquiry can lead. In doing so, this study proposes other ways of conceptualizing what a research process may become.

\section{Key words:}

Post-qualitative; inquiry; post-humanism; new materialism; rhizome; new ontology.

\section{Résumé:}

Cet article tente de situer une certaine généalogie de quelques mouvements qui sont liés à l'approche dite post-qualitative en recherche. Ces mouvements émergent en réponse aux tentatives, depuis les années 1990, de positiver, discipliner et objectiver la recherche qualitative par des normes et des rubriques qui établissent les voies à suivre tant dans la formation des chercheurs que dans la conceptualisation et la pratique de la recherche. Cette tendance, dans le cas des États-Unis où elle prend de plus en plus d'importance, est réaffirmée lorsqu'en 2002, le Conseil national de recherches publie un document qui établit comment la recherche scientifique en éducation doit être reproductible, généralisable, empirique et de préférence expérimentale. Cette décision s'inscrit dans une gouvernance néolibérale qui cherche à établir une vision unifiée de ce que devrait être la réalité et du rôle que la recherche devrait jouer à cet égard. Ce mouvement, qui se nourrit de différents référents et se projette dans différentes directions, questionne avec arguments les fondements onto-epistémologiques-méthodologiques et éthiques qui régissent cette tentative normative, tout en évitant les façons de faire de la recherche qui prédéterminent les sens ouverts et imprévisibles de tout processus de recherche et propose d'autres manières de conceptualiser ce que peut devenir un processus de recherche.

\section{Mots clés:}

Post-qualitatif; enquêter; post-humanisme; nouveau matérialisme; rhizome; nouvelle ontologie.

Fecha de recepción: 22-11-2018

Fecha de aceptación: 15-5-2019 


\section{Introducción}

Hace cuatro años (2014), en el marco de un seminario en el postgrado de Artes y Educación, Laura Trafí, que había seguido este programa y que entonces era profesora en la universidad de Milwaukee-Wisconsin, en Estados Unidos, nos presentó una investigación que había hecho con niños y niñas, quienes narraban, mediante filmaciones en vídeo, sus tránsitos en diferentes espacios en el camino hacia sus casas. Su presentación nos sorprendió porque no hablaba de análisis, ni de resultados. Apuntaba conceptos como devenir, desplazamientos, ... Le preguntamos qué marco ontológico y epistemológico fundamentaba su trabajo. Nos dijo que trataba de seguir lo que algunas autoras denominaban como 'nueva ontología'. Ante nuestra demanda de que explicara ese referente, nos dijo que no nos podía decir mucho porque era complejo y lo estaba explorando. Al terminar el seminario, algunos estudiantes de doctorado plantearon su interés por llevar a su formación lo que nuestra invitada había anunciado. Como una característica del programa de doctorado en Artes y Educación es que la formación se decide de manera conjunta, en el primer trimestre del nuevo curso (2015-16), realizamos un seminario sobre "investigación post-cualitativa", basado en artículos que se habían publicado en 2013 en el Journal of Qualitative Studies in Education y en el International Journal of Qualitative Studies in Education. Además de en el libro de Jackson y Mazzei (2012) en el que se nos invitaba a hacer investigación con la teoría. En el texto de presentación de este seminario, además de introducir el sentido de las diferentes lecturas que se ofrecían para su estudio y debate, Fernando Hernández (2015), uno de los autores del presente artículo, escribió

lo que vamos a explorar en este seminario nos planteará preguntas de profundo calado, no solo sobre los supuestos (ontológicos y epistemológicos) que guían la investigación -lo que significa investigar-, sino que también pueden cuestionar nuestras propias investigaciones. Esto no significa que tengamos que cambiar radicalmente nuestros supuestos ni el camino que estamos siguiendo en las tesis. Aunque quizá sí nos ayude a revisar algunas perspectivas que damos por sabidas y haga emerger algunas preocupaciones (y riesgos) en las decisiones que tomamos y que, a veces, camuflan tránsitos poco claros. Por ejemplo, investigaciones que se ponen bajo el paraguas construccionista, pero que en realidad se orientan por epistemologías positivistas (p1.) 
La perspectiva post-cualitativa en la investigación educativa: genealogía, movimientos, posibilidades y tensiones

Fernando Hernández-Hernández y Beatriz Revelles Benavente

En este curso académico tuvimos un seminario sobre 'Pedagogies of unknown' con Dennis Atkinson (Atkinson, 2011, 2018), con quien pudimos abordar su aproximación a algunos autores y conceptos clave en esta 'nueva ontología relacional'. Además de poder trasladarlos y vincularlos con la educación escolar y las artes. A este seminario siguieron, el curso siguiente (2016-17), otros dos, sobre 'nuevos materialismos' y 'nuevos empirismos', que contaron con la presencia de Beatriz ReveIles, la co-autora de este artículo, quien se había incorporado al grupo de investigación Esbrina (http://esbrina.eu/es/inicio/) como investigadora post-doctoral Juan de la Cierva. Ella aportó un bagaje de estudio e investigación, además de una experiencia de formación con Braidotti, van der Tuin y algunas de las autoras que estaban agitando, desde marcos post-feministas y post-humanistas, el hasta entonces más o menos plácido mar de la investigación en general y de la investigación cualitativa en particular. Al curso siguiente (2017-18), el seminario sobre 'políticas y pedagogías de los afectos' coordinado también por Beatriz Revelles, junto al de una introducción a la 'investigación rizomática' presentado por Paola Villanueva, quien en su tesis doctoral (Villanueva, 2017) había tomado la noción-invitación de Deleuze y Guattari (1980/2010) para plantear otra manera de fundamentar y llevar a cabo su investigación, nos permitió seguir profundizando en el camino iniciado. Ambas introdujeron y ampliaron los referentes onto-episte-metodológicos y éticos que iban dando sentido y contribuyendo a explorar lo que nos apuntó Laura Trafí unos años antes. Este curso (2018-19) lo hemos comenzado con un seminario de profundización en la perspectiva rizomática.

Pero ha sido, sobre todo, la orientación de la investigación "APRENDO: Cómo aprenden los docentes: implicaciones educativas y retos para afrontar el cambio social" (EDU2015-70912-C2-1-R)², de la que se dará cuenta en varios de los artículos que componen este monográfico, lo que nos ha permitido poner en cuestión algunas de nuestras concepciones sobre lo que puede significar investigar y dar cuenta (escribir) de una investigación en educación - y en ciencias sociales. Este enfoque, que vamos a denominar como 'post' en este artículo, contiene y promueve diferentes movimientos y afronta diversas problemáticas, no lo tomamos como una nueva moda a seguir, ni como una invitación a poner el contador a cero. Por eso, invitamos a quienes nos lean a tener en cuenta sus

2 https://esbrina.eu/es/portfolio/apren-do-como-aprenden-los-docentes-implicacioneseducativas-y-retos-para-afrontar-el-cambio-social/ 
planteamientos, en la medida en que generan desafíos, pero también interrogantes y necesarias críticas. Por eso tratamos de estar abiertos a lo que cuestiona y amplía nuestras posicionalidades (Hernández-Hernández y Sancho-Gil 2015), consideradas siempre como estratégicamente provisionales y que nos abren caminos para seguir aprendiendo juntos. Esta finalidad orienta lo que planteamos en las siguientes páginas, en las que tratamos de presentar una cierta genealogía de estos 'giros' que plantean cuestionamientos a la centralidad del sujeto y la subjetividad en los estudios cualitativos, así como a la interpretación (o comprensión) como perspectiva o sentido del abordaje cualitativo.

\section{Un mapa para esbozar un territorio (aunque el mapa nunca es el territorio)}

The 'posts' announce a radical break with the humanist, modernist, imperialist, representationalist, objectivist, rationalist, epistemological, ontological, and methodological assumptions of Western Enlightenment thought and practice (St. Pierre, 2011, p. 615).

Hay una pintura que Gauguin realizó en 1897, durante su segunda estancia en Tahití, que tituló 'De dónde venimos, quiénes somos, adónde vamos', que nos sirve como punto de partida para lo que es la finalidad principal de este artículo. Tratar de esbozar una cierta genealogía de las diferentes nociones, campos, movimientos y debates que se pueden agrupar bajo el paraguas de lo post-cualitativo. Afrontamos esta tarea siendo conscientes, como señala Jon Meacham (2017), que "One of the great historical questions is also one of the most difficult to answer: When, precisely, did a given phenomenon, cultural or political, truly begin?" (p.21). A lo que añadimos, que tratar de 'ordenar' los diferentes planos de 'lo post' será siempre una tarea provisional, puesto que

"each researcher who puts the 'posts' to work will create a different articulation... remix, mash-up, assemblage, a becoming of inquiry that is not a prio$r i$, inevitable, necessary, stable, or repeatable but is, rather, created spontaneously in the middle of the task at hand, which is always already and, and, and... (St. Pierre, 2011, p. 620, énfasis en original). 
La perspectiva post-cualitativa en la investigación educativa: genealogía, movimientos, posibilidades y tensiones

Fernando Hernández-Hernández y Beatriz Revelles Benavente

No resulta, por tanto, tarea sencilla saber, como apunta Meacham en la cita anterior, cuándo comienza el movimiento 'post-cualitativo'. Tampoco si hay un acontecimiento específico que lo desencadena. Pero es que tratar de buscar un punto cero sería, por otra parte, contradictorio a un enfoque, perspectiva o movimiento, que cuestiona por su artificiosidad la lógica de la causalidad lineal. Asumamos pues, de partida, que transitamos entre conversaciones y derivas. Que pensar lo 'post' contra algo no tiene sentido, pues ese algo es lo que nos permite pensar desde otro lugar, pues sin su presencia nuestro 'nuevo' pensamiento no sería posible. Dado que no estamos en el origen de nada, sino en una conversación que se nutre de otras conversaciones y referentes que se mueven y cruzan en múltiples direcciones. Lo que también nos lleva a asumir que, como señala Higgins (2017), "The mo(ve)ment of post-qualitative research ... inherits its historicities: both actual and virtual" (p. 98). Por eso, comencemos, sin ánimo de ser exhaustivos, por señalar algunos movimientos que son concomitantes al despliegue de los flujos de un pensamiento post-cualitativo, conscientes, como apunta St. Pierre, que es difícil escapar a nuestras herencias: "Even those who've studied the 'posts' and, in particular, poststructural theories of subjectivity, seem unable not to write the humanist human being" (St. Pierre, 2011, pp. 619-620, énfasis en original). Y lo hacemos a partir del diálogo con algunas de las autoras que han ido contribuyendo a constituir la genealogía y tensiones de lo post-cualitativo. El propósito de esta corriente de propuestas -en el caso de que podamos hablar de propósitos- es celebrar las diferencias metodológicas y la complejidad de la investigación cualitativa y abogar por una mayor apertura, imaginación y toma de riesgos, especialmente para llevar a cabo una investigación que se base en las "post-teorías". Estas nuevas ontologías exigen desplazamientos metodológicos, accidentes, saltos conceptuales y deslizamientos, así como vinculaciones teóricas. Como han argumentado Lather (2007), Koro-Ljungberg, et al, (2009), Jackson y Mazzei (2012) y Koro-Ljungberg, (2015) las teorías (lo que guía y fundamenta una investigación) y las metodologías están interconectadas para posibilitar relaciones prácticas. Además, pueden ser consideradas como movimientos políticos contra la ciencia normativa, especialmente entre aquellos académicos interesados en ontologías emergentes y metodologías sorprendentes $Y$ es que, cuando las metodologías son consideradas como inmanentes, cambiantes y transformadoras (Deleuze y Guattari, 1991/1993) -y portadoras de elementos desco- 
nocidos e imprevistos-, las prácticas de investigación parecen acercar a los académicos a la apertura y la imaginación. Las publicaciones que se vinculan a esta línea tratan de revisar, expandir y examinar críticamente el estado de la investigación cualitativa normativa en la medida en que se ha configurado como un espacio de pensamiento que tiende a eludir toda duda. Algo que, por otra parte, es inherente a cualquier proceso de investigación. En la investigación humanística cualitativa convencional (St. Pierre, 2011) los referentes metodológicos y los métodos que les conectan con la realidad son a menudo considerados como relativamente simples y concretos, comparados con las entidades más complejas o abstractas que ayudan a generar conocimiento, conceptos y argumentos de manera diferente. Este marco permite pensar el sentido de la investigación desde otra perspectiva, con otros fundamentos y propósitos. Pero sobre todo con una abertura a la imaginación que permita pensar que investigar no es sólo seguir un camino prefijado, en el que se aplican unos métodos, para dar cuenta de unos resultados que ya se preveían en las preguntas iniciales.

Para situar cómo se han ido gestando estos modos de pensamiento, comencemos situando algunos de los movimientos hacia una investigación post-cualitativa, tomando como referencia la caracterización que hace Patti Lather (2013) de cómo se ha ido constituyendo una ontología relacional post-cualitativa. Estas fases no son correlativas, sino que se superponen y relacionan ${ }^{3}$.

El punto de partida podría ser una investigación interpretativa convencional que surge de una visión humanista y que proyecta un sujeto que se supone tiene una voz auténtica y genuina. Esa voz y las acciones de ese sujeto son las que posibilitan (se supone) realizar descripciones transparentes de las experiencias vividas, al tiempo que se asume la creencia de que los métodos que facilitan esas descripciones pueden acercarnos a la verdad de la experiencia de los sujetos. En otro lugar (Hernández y Rifà, 2011) hemos señalado que, durante la década de los noventa, se configuró una perspectiva de investigación narrativa, considerada como una modalidad de investigación cualitativa que, en un sentido amplio, se basaba en narrativas que describían la acción humana (Casey, 199596). Se comenzó entonces a utilizar el término 'investigación narrativa', que adquirió un carácter fundacional, a partir del artículo que Conelly

3 Las referencias que hemos incorporado son solo ilustrativas, puesto que un recorrido exhaustivo por estas fases daría para otro artículo o para un libro. 
La perspectiva post-cualitativa en la investigación educativa: genealogía, movimientos, posibilidades y tensiones

Fernando Hernández-Hernández y Beatriz Revelles Benavente

y Clandinin (1990) publican en Educational Researcher, en el que destacan una doble influencia en esta perspectiva, relacionada con cuestionamiento -por su insuficiencia- del paradigma positivista/realista a la hora de captar las experiencias de vida de los seres humanos, y con la deriva que adopta la investigación en ciencias sociales hacia una investigación que recoja y de sentido a esas experiencias. Esto da paso a una fecunda línea de investigación (Clandinin y Conelly, 1995), que se articulan en lo que Denzin (1997) denominó narrativas del yo, en la que quien investiga forma parte y aparece en un hilo narrativo, que no se impone al lector, sino que le deja lugar, para que establezca sus propios nexos y relaciones (Conelly y Clandinin, 2000). Este movimiento permite "alterar y cuestionar las normas del discurso científico que se consideran incuestionables a partir de destacar la experiencia vivida, los detalles íntimos, la subjetividad y las perspectivas personales" (Ellingson y Ellis, 2008: 450). Pero este enfoque no tiene en cuenta que la centralidad en la voz y la experiencia de los sujetos plantea problemas no afrontados sobre el sentido androcéntrico de la subjetividad y la experiencia, la interpretación que de ella realiza quien investiga y la ontología y epistemología humanista que lo guía.

En otra fase, la investigación cualitativa reconoce múltiples realidades, voces, textos que se recogen y presentan de manera desordenadas. La centralidad ya no está en la subjetividad de quien da cuenta de su experiencia, sino en las relaciones que la posibilitan. Se introducen nociones como reflexividad, diálogo, empoderamiento, etc., pero permanece dentro del marco onto-epistemológico humanista, que se fundamenta en los conceptos humanistas de lenguaje, realidad, conocimiento, poder, verdad, resistencia y sujeto. La noción de reflexividad (Steier, 1991; Macbeth,2001; Kincheloe y Berry, 2004) adquiere un papel clave en la medida en que lo que da sentido a una investigación narrativa es la capacidad del investigador para dar cuenta, de manera minuciosa y exigente de lo que fundamenta las decisiones que va tomando y de las relaciones que establece en el proceso de escritura. De esta manera el campo de la investigación cualitativa se centra, se disciplina, se regula y se normaliza a medida que los manuales cualitativos (Brizuela et al. 2000), los libros de texto y las revistas aparecen 'momentos' y 'diseños', y se fija el 'proceso de investigación' (Kohler-Riessman, 2008) y su nomenclatura (Schwandt, 1997). De este de modo es posible conocerlo de antemano para ofrecer una secuencia de cursos sobre la investigación cualitativa, 
enseñar a alguien a 'hacer investigación fenomenológica' y, de manera especial, cómo enseñar a alguien a analizar los datos (Saldaña, 2009). Pero ambas operaciones, la determinación de antemano del curso de la investigación y la relación con los datos como algo que está ahí fuera y que quien investiga ha de encontrar para darles sentido, no tiene en cuenta como el investigador está presente en aquello que investiga, ni con el sentido impredecible de todo proceso de investigación.

En otro momento se asumen teorías posmodernas (Cherryholmes, 1988; Packwood y Sikes, 1996; Scheurich, 2001; Cooper y White, 2012) para plantear conceptos asociados a la investigación cualitativa como validez, voz, datos, empatía, autenticidad, experiencia, entrevistas, el campo, reflexividad, claridad, etc. (Denzin y Lincoln, 2002, 2005). Sin embargo, esta perspectiva se encuentra, en opinión de Lather (2013), estancada desde hace años, en la medida en que los investigadores cualitativos, que van sustituyendo la noción de investigación por la de indagación (inquiry), recurren a la defensa no sólo de la metodología, sino también de las diversas epistemologías que les sustentan (teorías feministas, de raza, de clase, posmodernas, postestructuralistas etc.). Y lo hacen sin tener en cuenta, como señaló la revisión de Koro-Ljungberg et al. (2009), que la investigación en educación elude la coherencia entre los fundamentos onto-epistemológicos, y la perspectiva metodológica que se adopta. Además de dar una primacía determinista a la orientación metodológica que se dice adoptar (Koro-Ljungberg, 2015). Lo que hace que el campo de la investigación cualitativa siga estructurado como si fuera un todo ordenado, y que "métodos mixtos interpretativos" se comenzaran a normalizar (Tashakkori y Teddlie, 2003; Jonson y Onwuegbuzie, 2004; Creswell et alt. 2006; Mason, 2006; Greene, 2008) como indicios de la domesticación via metodología de procesos que fluyen en diferentes direcciones.

Finalmente, la perspectiva post-cualitativa se comienza a articular cuando tiene lugar un movimiento que se agita en torno al concepto de 'becoming', a partir del sentido que le dan Deleuze y Guattari (1980/2010) y que Barad desarrolla cuando se refiere a lo que sucede en un proceso de investigación

In an important sense, this story in its ongoing (re)patterning is (re)(con)figuring me. ' $\mathrm{I}$ ' am neither outside nor inside; ' $\mathrm{I}$ ' am of the diffraction pattern. Or rather, this ' $\mathrm{I}$ ' that is not ' $m e^{\prime}$ alone and never was, that is always already 
La perspectiva post-cualitativa en la investigación educativa: genealogía, movimientos, posibilidades y tensiones

Fernando Hernández-Hernández y Beatriz Revelles Benavente

multiply dispersed and diffracted throughout spacetime(mattering), including in this paper, in its ongoing being-becoming is of the diffraction pattern (2014, pp. 181-182).

Esta orientación hacia el becoming (devenir, fluir,...) se genera cuando, como señala Lather, las investigadoras, cansadas de una década (la del milenio) de defender la investigación cualitativa, vuelven a imaginar y realizar una investigación que podría producir conocimientos diferentes. Esta investigación no puede ser descrita ordenadamente en los libros de texto o manuales. No existe un enfoque y instrumento metodológico que se pueda aprender sin problematizarlo. Desde este movimiento, se comienza a hacer investigación de manera diferente, pues se adopta una onto-episte-medología y una ética que asume que

"[i]ndividuals do not exist as fixed or permanent entities separate from their surroundings but as ongoing relations of becoming in a world that is also always becoming" (Atkinson, 2015, p. 44).

Es desde este movimiento que el término y el enfoque "post-cualitativo" comienza a tener cierto sentido (Lather, 2013, p. 634-635).

\section{La reacción ante la imposición neopositivista}

Pero si esto es lo que sucede desde el interior del campo de la investigación cualitativa, no se puede perder de vista que, durante este tiempo, también se producen movimiento que buscan disciplinar la investigación cualitativa mediante estándares y rúbricas. En una época marcada por la lógica neoliberal como la que nos habita, Miñana y Rodríguez (2003), señalan que "Lo característico del neoliberalismo es proponer una visión economicista, ligada a la primacía del mercado como la única legítima para orientar las decisiones en el campo educativo, y considerar la educación como mercancía" (p.7). Este contexto afecta de manera evidente a la investigación en educación y en particular a la investigación cualitativa. Patti Lather (2013) apunta algunos indicadores de cómo se ha ido fraguando en Estados Unidos una tendencia hegemónica, que se ha proyectado en otros países, donde nuevos positivismos reclaman una reelaboración de la objetividad como prueba de refutabilidad, haciendo 
que los criterios de demarcación de la ciencia sigan siendo normativos en lugar de descriptivos.

Esta línea se articula, de manera especial, cuando las concepciones que fijó el Empirismo lógico (St. Pierre, 2016) penetran en la investigación cualitativa, después de los giros interpretativos y lingüísticos, sobre todo a partir de las acciones que toma la National Science Fundation, durante la presidencia de George Bush, para normativizar, mediante las directrices que se establecen en los Scientific Principles for Education Research (2002), el tipo de investigación que se ha de realizar y, por tanto, legitimar con ello solo un determinado tipo de investigación. O lo que es lo mismo, se fija desde qué fundamentos onto-episte-metodológicos y éticos se ha de investigar. Estos movimientos contrastan con el posicionamiento impulsado desde la Asociación Americana de Investigación Educativa (AERA, por sus siglas en inglés) quien había presentado una serie de estándares para las "ciencias sociales empíricas" (AERA, 2006) que incluye criterios más cualitativos y amigables dentro de un compromiso con la transparencia de la lógica de la investigación. Esta influyente asociación volvió a presentar unos años después un conjunto de normas para la "investigación orientada a las humanidades" (AERA, 2009) en los que se habla de disonancia, incomodidad y reflexividad, fronteras borrosas entre las humanidades y las ciencias sociales, y espacio para imágenes evocadoras y narrativas. El documento ofrece un espacio para "apartarse de las ortodoxias" en las demandas de transparencia y rechazo directo tanto del movimiento hacia los "abstractos estructurados" como de la neutralidad a favor de la investigación que ilumina, critica y evalúa.

En resumen, la disputa sobre la ciencia que puede proporcionar evidencias para la práctica y la política enfrenta el positivismo rescatado por el neoliberalismo contra una comunidad cualitativa en riesgo de asimilación. Lo que se propone es reducir lo cualitativo a un instrumentalismo que satisfaga las demandas de la cultura de la auditoría (Lather, 2013, p. 636). Esto lleva a Lather y St. Pierre, junto a otras autoras como De Freitas, Jackson, Mazzei, Koro-Ljungberg, Richardson, ... a considerar que esta imposición supone un retroceso, en nombre de la insistencia en la importancia de la lucha tanto epistemológica como ontológica, en el gobierno de la investigación, y de llamar la atención sobre la forma en que el conocimiento basado en la investigación es conceptualizado y producido, a explorar la ontología que se les impone, y que muchos investigadores cualitativos terminan adoptando. En su recorrido se pro- 
La perspectiva post-cualitativa en la investigación educativa: genealogía, movimientos, posibilidades y tensiones

Fernando Hernández-Hernández y Beatriz Revelles Benavente

ponen pensar y moverse más allá de la ontología humanista (St. Pierre, 2014). Esto las lleva a revisar nociones como la igualdad, la representación, la voz, la experiencia, el yo, el análisis, los datos, la lógica binaria, la entrevista, la reflexividad y muchos otros conceptos centrales de los marcos onto-epistemológicos neopositivistas e interpretativos. Conceptos y prácticas que se borran y a veces se evitan por completo o se consideran irrelevantes. A todo ello se une el interés por lo material en las relaciones entre los individuos y los mundos materiales que les conforman (Barad, 2007; MacLure, 2013; Dolphijn y van der Tuin, 2012).

Lather (2013), por su parte, propone que los ejemplos de investigación post-cualitativa utilicen la multidireccionalidad y nociones como cuerpos post-humanos, redes, alteridades y disparidades. En estos planteamientos, unos autores que son inspiradores del giro post-cualitativo son Deleuze y Guattari. Especialmente el primero (Coleman y Ringrose, 2013). De esta manera, en lugar de estandarizar la investigación cualitativa en nombre de proporcionar mejores garantías probatorias, estos esfuerzos han dado lugar a una "sorprendente reafirmación de los enfoques cualitativos", en los que las normas compartidas y la distinción entre hechos y valores se mantienen bajo sospecha (Weyland, 2005, p. 392).

\section{Señalar otros movimientos}

En los anteriores párrafos hemos esbozado el cruce en los intentos de normativizar la investigación en educación y sus efectos en la positivización de la investigación cualitativa, al tiempo que esta perspectiva era afectada por una serie de cambios y movimientos. Pero en otros círculos también se planten debates sobre el sentido de la investigación, la relación entre lo humano y lo no humano. Para dar cuenta de forma breve de este panorama, que también influye en el movimiento post-cualitativo, hemos considerado algunas entradas de la ordenación realizada por Judit Onsès (2018) en su tesis doctoral, en la que presenta algunos de los planos en los que se articula y refleja lo post, y que nosotros hemos completado con otras lecturas y referencias. Todo con la voluntad de ofrecer a quien lea este artículo un punto de partida para situar y explorar otras vías en relación con la génesis y consecuencias de lo post en la investigación en educación. Si bien, como señala Onsès (2018), la mayoría de 
textos comienzan a publicarse en la primera década del siglo XXI-, es posible detectar algunos antecedentes vinculados a algunos 'malestares' que afectan a quienes realizan investigación cualitativa en algunos ámbitos de las ciencias sociales:

El malestar que reflejan algunos movimientos feministas y postcoloniales con relación al frecuente propósito e interpretación de la Historia con intención de reflejar una realidad única, verdadera y hegemónica y que se refleja también en el canon científico que sustenta las diversas disciplinas de conocimiento. Estos colectivos consideran que este relato no les representa, porque está contada desde un único punto de vista: la versión de los vencedores, que coincide con el hombre blanco occidental colonizador. Por lo que la Historia -y por extensión el mundo- no la hacen las personas, sino una franja concreta de personas, y la hacen a su medida. Y eso coincide con la perpetuación de unas Humanidades que toman al hombre como centro del universo, donde la Historia ha excluido o minusvalorado el papel de las mujeres en ella. Hay una reivindicación para que la Historia sea explicada por varias voces y no sólo desde la del Hombre. Y esto conecta con el desvelamiento de las relaciones de poder: quien escribe la Historia es quien tiene el poder (Haraway, 1991, 1997/2004; Braidotti, 2013/2015; Revelles-Benavente, 2017).

La necesidad de cuestionar la ontología humanista para plantear una alternativa a la metodología cualitativa humanista. Una serie de pensadoras (Haraway, 1988, 1991; Braidotti, 2015; Revelles-Benavente, 2018) que beben del post-estructuralismo y ponen sobre la mesa la crisis del humanismo heredero de la Ilustración, toman el pensamiento dualista de Descartes como metáfora de racionalidad ${ }^{4}$, la representación y el pensamiento binario y dicotómico, basado en verdades y valores universales, centrada en el cogito, en el sujeto que sabe, y donde lo humano no sólo está en el centro de todas esas categorías de indagación cualitativa, sino antes de ellas. De aquí el reflejo que esta ontología humanista tiene en

4 Si como nos ha señala Donna Haraway (1988), el conocimiento es siempre contextual y situado, sería importante no olvidar que Descartes tuvo que sortear en esa representación dualista que se le ha criticado, la presencia de la Inquisición -que había condenado a Galileo- y la dificultad para escapar de su control, a la hora de manifestar el pensamiento de alguien que se consideraba un fiel católico. Con todo, no hay que olvidar, por otra parte, que después de la publicación en 1649 de "Les Passions de l'Ame", Descartes se plantea cómo el cuerpo y la mente-alma se relacionan entre sí, cómo algo material —el cuerpo- interactúa con algo inmaterial —la mente-alma y que se articulan en la glándula pineal. 
La perspectiva post-cualitativa en la investigación educativa: genealogía, movimientos, posibilidades y tensiones

Fernando Hernández-Hernández y Beatriz Revelles Benavente

las categorías que hemos inventado para organizar y estructurar la metodología cualitativa humanista: problema de investigación, preguntas de investigación, revisión de la literatura, métodos de recolección de datos, análisis de datos y representación- asumen una profundidad en la que el ser humano es superior y se separa de lo material -Ego/Otro, sujeto/ objeto, y humano/no humano. Frente a ello, y por lo que vamos enunciando en esta genealogía, la actualidad pide otros modos de pensar, de hacer ciencia y filosofía. Estas y otras autoras problematizan, al tiempo que buscan alternativas en el sentido de la invitación que realizan Lather y St. Pierre (2013):

"If we cease to privilege knowing over being; if we refuse positivist and phenomenological assumptions about the nature of lived experience and the world; if we give up representational and binary logics; if we see language, the human, and the material not as separate entities mixed together but as completely imbricated "on the surface" - if we do all that and the "more" it will open up - will qualitative inquiry as we know it be possible? Perhaps not. (p. 630).

Reconocer estos 'if' supone reconocer que el autor existe antes de la escritura, por lo que el investigador puede escribir una propuesta de investigación que describa lo que hace antes de comenzar su proceso de indagación. Esta suposición asume que en realidad hay un principio, un origen. En lugar de considerar que quien investiga siempre se está enredada (entanglement) en una trama que le antecede y que interviene en el proceso de la investigación. Esta situación de enredo lleva a Lather y St. Pierre (2013) a cuestionar el sentido que se le da a la investigación cualitativa:

Entanglement makes all the categories of humanist qualitative research problematic. For example, how do we determine the "object of our knowledge"the "problem" we want to study in assemblage? Can we disconnect ourselves from the mangle somehow (Self) and then carefully disconnect some other small piece of the mangle (Other) long enough to study it? What ontology has enabled us to believe the world is stable so that we can do all that individuating? And at what price? How do we think a "research problem" in the imbrication of an agentic assemblage of diverse elements that are constantly intra-acting, never stable, never the same? (p. 630) 
El giro afectivo ${ }^{5}$. Que se vincula, como señalan Ali Lara y Giazú Enciso Domínguez (2013), a lo que Greco y Stenner (2008) intuyeron como un interés de algunos académicos por la 'emocionalidad de la vida pública' y de "las instituciones, sectores y subsistemas que la conforman" y que supone "cambio en la concepción del afecto que ha venido a modificar la producción de conocimiento y la lógica misma de las disciplinas" (Lara y Enciso Domínguez, 2013, p. 102). En los antecedentes de este movimiento, se pueden localizar, por una parte, en el artículo The Autonomy of Affect de Brian Massumi (1995) quien toma como referente a Deleuze ${ }^{6}$, vinculando el afecto y su autonomía a la voluntad de escapar del 'confinamiento' discursivo (Massumi, 2002, p. 228). El segundo referente será el artículo de Sedgwig y Frank (1995) Shame in the cybernetic fold, que dialoga con el trabajo del psicólogo Silvan Tomkins (1963; 1991) Affect, Imagery, Consciousnnes, donde se pone el énfasis en los conductores corporales que propician los movimientos afectivos. Otras autoras (Cvetkovich, 2012; Hickey-Moody, en prensa), consideran que los precedentes de la teoría afectiva se sitúan más bien en algunas pensadoras feministas que durante los noventa centraban su eje onto-epistemológico en reconfiguraciones del cuerpo y como éste era afectado ya que los afectos se definen como el aumento o disminución de la capacidad de un cuerpo para actuar (Hickey-Moody, en prensa). A partir de aquí se articularán dos pilares clave del pensamiento post: la rebelión ante 'el imperialismo discursivo' (Grecco y Stenner, 2008) y el énfasis en las capacidades del cuerpo, tal y como señala Patricia Clough (2008) para afectar y ser afectado. Ambas líneas, la del pragmatismo de Tomkins, y la de los 'vuelos subjetivos' de Deleuze se expanden a través de Sedgwick y Massumi y se trenzan en la perspectiva post. Sin olvidar que el interés por el cuerpo se conecta con la obra de Spinoza (1667/1980), especialmente a través del diálogo que Deleuze establece con su obra (Deleuze, 1968/1996;1981/2009). La influencia del giro afectivo en la ontología post-cualitativa se puede observar en Latter (2013), cuando señala que

5 Asumimos que esta entrada es una indicación que debe mucho al trabajo sobre el Giro Afectivo de Ali Lara y Giazú Enciso Domínguez( 2013). Quienes se interesen por esta temática también pueden acercarse a un texto sobre la precuela del giro (Enciso Domínguez y Lara (2014), el artículo de Ali Lara (2015) en torno a algunas de las fuentes filosóficas de este movimiento y al texto de Vinciane Despret (2004) sobre el lugar de los afectos antes del giro afectivo.

6 Massumi había realizado la traducción al inglés del libro de Deleuze y Guattari 'Mil Mesetas: Capitalismo y Esquizofrenia' en 1987, obra que se convertirá en uno de los goznes básicos que guiarán algunas de las proposiciones del movimiento post. 
La perspectiva post-cualitativa en la investigación educativa: genealogía, movimientos, posibilidades y tensiones

Fernando Hernández-Hernández y Beatriz Revelles Benavente

Another kind of researcher subjectivity is also called for. Affect theory might provide some direction as it is, in Berlant's argument, a new phase in ideology theory (2011, p. 53). Berlant's take on affect theory troubles "the liberal culture of true feeling" (p. 65) that is so sentimentally present in much of the qualitative research about the "vulnerable ethnographer" (Behar, 1996) and autoethnography. (p. 640).

Estos afectos se convierten precisamente en los generadores de conocimiento y las fronteras onto-epistemológicas de un objeto de investigación dado, teniendo en cuenta que dichos objetos de investigación carecen de límites establecidos por el o la investigadora, ya que como define Hickey-Moody (en prensa), "affect can be transgressive: leaking between bodies and ideas and showing up the edges of 'thought'." Como veremos a continuación, esto ha supuesto dos grandes tensiones para las comunidades epistemológicas previamente mencionadas, ya que altera la definición de 'agencia' pasando de algo que alguien puede poseer a una 'agencia' que se materializa durante la relación (Barad, 2007; RevellesBenavente, 2017). Por lo que la transformación social también reside en el afecto (Colman, 2010) y no en una indentidad política individual, sino que se transforma en una intra-seccionalidad (van der Tuin y Dolphijn, 2013). La segunda transformación que veremos es el desbordamiento de los límites espaciotemporales geográficos que tienen que ver con la capacidad de delimitar espacios físicos y lógicas lineales de causa y efecto (Barad 2010; Revelles-Benavente, 2015). Por su parte, esta relacionalidad es la que define en mayor medida la pedagogía afectiva que abordamos desde nuestros seminarios de doctorado y de máster ya que, siguiendo a Anna Hickey-Moody (2016: 264) entendemos dicha pedagogía como una reconfiguración corporal y una geografía emergente cultural de los sentimientos humanos, lo cual se enmarca como una mediación material como intercambio pedagógico.

El replanteamiento del sentido de la metodología. La orientación metodológica que se propone dentro de estos giros onto-epistemológicos se refiere a una metodología que se considera dentro de un entretejido en el que ontología y epistemología son parte de un mismo plano de análisis (Barad, 2007, van der Tuin, 2015). En este sentido, consideramos que estas tres dimensiones del conocimiento intra-accionan a su vez con la parte ética del conocimiento (Revelles-Benavente, 2010). Por lo tanto, la metodología es la posición situada de un investigador o investigadora 
dentro de su investigación, es la relación en sí misma del investigador o investigadora con el objeto de investigación hacia un futuro que se entiende como afectivo (Coleman, 2017); es decir, como una sociología de los sentimientos que abre posibilidades para una transformación social.

Esta metodología es radicalmente situada lo que hace que una traducción literal que pueda adoptar la metodología de una investigación a otra sea la pista que tenemos para comprobar que una investigación no está deviniendo en unos procesos óptimos para los resultados de la misma. Es decir, desde una perspectiva post-cualitativa es imposible diseñar una metodología previamente a la relación investigadora por lo que aplicar directamente cualquier diseño previo estaría imponiendo una serie de corsés onto-epistemológicos que oprimen el dinamismo del objeto propio de investigación. Hay unas guías o pautas, claro está, que conforman la propia visión del o la investigadora.

Las metodologías que proponen los giros post-cualitativos tienen, definitivamente, algunos aspectos en común. Rebecca Coleman (2017, p. 528) define una metodología innovadora e inventiva basada en el afecto a través de Gabrielle Ivinson y Emma Rennols de la siguiente manera como una exploración del "affect associated with everyday practices through the multiple activities, rituals and routines that comprised the micro-intensities of everyday life', and offer their participants 'ways to experience, think and imagine futures differently from those afforded by the community's mining past' (2013: 374)." Estas metodologías documentan, prueban, provocan y estimulan la imaginación invocando un futuro sensorial en el que se materializa lo especulativo y lo performativo, la acción y la invención (ibid, p. 539).

Hacia una ontología y una política relacional. Así pues, cuando pensamos en organizaciones del conocimiento relacionales, la materialización de una política también relacional aparece. Implícitamente se ha ido dibujando a lo largo de este artículo esta ontología y política relacional, que concuerda con una ética que se materializa a través del afecto y la ausencia de las jerarquías, más que como un acto de respeto o tolerancia para con el "otro". Según Spinoza (1667/1980), los afectos positivos y negativos pertenecen indistintamente al ámbito de la mente; lo que los diferencia es que un aspecto positivo incrementa la capacidad de actuar y un afecto negativo la reduce (Hickey-Moody, en prensa). La interpretación de este marco se ha dividido en dos vías que, aunque podrían complementarse tienen repercusiones drásticamente distintas 
La perspectiva post-cualitativa en la investigación educativa: genealogía, movimientos, posibilidades y tensiones

Fernando Hernández-Hernández y Beatriz Revelles Benavente

para la interpretación política del afecto. Por una parte, hay pensadoras feministas que han relacionado esta división activa y pasiva mediante la enfatización de afectos alegres y tristes (Camps, 2011) que materializan determinadas huellas históricas que producen el conocmiento de los mismos (Bargetz, 2014). Esta interpretación es bastante útil sobre todo cuando intentamos que dichos afectos se conviertan en unidades de análisis radicalmente empíricas (Clough, 2009) que expliquen determinados fenómenos socioculturales (Revelles-Benavente, 2017). Sin embargo, explicar los afectos desde este incremento o disminución activo implica poder explicarlos desde el movimiento, el deseo (Hernández, 2011), la transformación social (Colman, 2010), la encarnación sentimental geográfica (Hickey-Moody, en prensa), y; por supuesto, la "capacidad-derespuesta" (Revelles-Benavente y González, 2019) que se materializa en el nivel de la micropolítica. Esta capacidad de respuesta implica que, dado que nuestros cuerpos se transforman cuando se ponen en relación con otros factores (humanos y no humanos) y solo adquieren significado durante esa relación y no previamente (como parte de la intra-acción baradiana de la ontología relacional), la capacidad de acción política también se configura en esta relación. Por lo tanto, a pesar de que determinados afectos puedan materializarse según algunas huellas materialistas históricas (Bargetz, 2014; Berlant, 2011, Ahmed, 2010), centrarse en la transformación de la relación es lo que hace que determinados procesos se lleven a cabo siguiendo una ética feminista o de implicación social. Es decir, el afecto pone en práctica la capacidad del investigador o investigadora para aceptar riesgos (Harway, 2016) a nivel micropolítico sabiendo que tienen una repercusión automática en lo global.

\section{Lo post-cualitativo como posibilidad de una investigación otra}

Como hemos visto, desde hace tres décadas, el término "investigación post-cualitativa" ha ganado atención y ha servido como impulso para explorar nuevas direcciones onto-episte-metodológicas y éticas entre algunos académicos que venían desarrollando investigaciones bajo el amplio paraguas de la investigación cualitativa. Varias revistas han dedicado números especiales a la investigación post-cualitativa: en The International Journal of Qualitative Studies in Education (2013) editaron un número específico sobre "Post Qualitative Research"; en Cultural 
Studies $\leftrightarrow$ Critical Methodologies (2013) plantearon el cuestionamiento de la noción de "Data"; en Qualitative Inquiry (2014), seguramente la publicación que ha ido recogiendo con una mayor continuidad los movimientos de esta perspectiva, presentaron un número dedicado al tema "Data Analysis after Coding; y, de nuevo, en Cultural Studies $\leftrightarrow$ Critical Methodologies, (2016) un número dedicado al 'New Empiricism'. La AERA (American Educational Research Association), especialmente el SIG (Special Interest Group) en Qualitative Inquiry, acoge presentaciones que debaten sobre esta perspectiva. En los congresos de la ICQI (International Conference in Qualitative Inquiry) que se organizan desde hace 15 años en la Universidad de Urbana Champagne, con el especial liderazgo de Norman Denzin (responsable junto a Ivone Lincoln de las sucesivas ediciones del Handbook on Qualitative Research y autor del Qualitative Manifesto (Denzin, 2010), se acoge y da cuenta de los movimientos de esta perspectiva. Congreso que desde hace dos años también se celebra en Europa, como espacio de encuentro de quienes comparten un sentido de la investigación otra.

Sin embargo, la investigación que se adscribe a esta perspectiva es bastante desconocida (cuando no rechazada) en la investigación social, especialmente por parte de quienes se adscriben a una perspectiva neopositivista y estandarizada de la investigación. Aunque en este artículo no hemos pretendido realizar una completa genealogía que dé cuenta de todos los movimientos y tensiones que llevan iniciar un giro frente a lo que comenzaba a ser un sentido normativo por parte de la investigación cualitativa, queremos apuntar que 'lo post-cualitativo' no es una tendencia o perspectiva que tiene una única cara y que se articula en una única propuesta. Lo que comparten los autores y autoras es una vuelta a la 'realidad' que había sido subsumida en los textos y ponen en cuestión, como señala Onsès (2018) "las nociones de realidad, naturaleza, humanidades, el ser humano, y los modos de entender y hacer ciencia. Es por ello por lo que en algunos círculos académicos se habla de post-ontología, post-humanismo, nuevos materialismos e investigación post-cualitativa" (p. 81). Pero estas propuestas presentan tensiones y cuestionamientos, no solo por parte de quienes se acercan a ella desde posiciones de radical oposición a las mismas (ver texto de Lather, 2013), sino de quienes lo hacen desde el interés por comprender lo que estas autoras plantean en su afán por generar una visión del mundo social "that demands a different way of thinking about knowledge and research" 
La perspectiva post-cualitativa en la investigación educativa: genealogía, movimientos, posibilidades y tensiones

Fernando Hernández-Hernández y Beatriz Revelles Benavente

(Green 2013, p. 750). Quizá el principal cuestionamiento sea si lo que plantea la perspectiva post-cualitativa es realmente investigación o es ensayo filosófico ${ }^{7}$. Por eso, terminamos nuestro acercamiento a lo postcualitativo apuntando una serie de tensiones, que recoge Green (2013) y que nosotros hemos ampliado:

La pérdida de sistematicidad. Una de las problemáticas que se derivan de los planteamientos y de la terminología utilizada en la perspectiva post-cualitativa es la falta de una sistematicidad que permite una comprensión compartida, a la hora de explorar aquellos fenómenos a los que se presta atención en una investigación En este sentido, Green se plantea con preocupación si la pérdida del carácter sistemático de la investigación social, donde los sistemas (directrices, reglas, preceptos, suposiciones) son abiertos y disponibles para todos, puede llevar a que la investigación se vuelva menos distinguible de otros esfuerzos humanos importantes como la literatura y la creación en diferentes ámbitos.

La pérdida de lo cognitivo. La perspectiva post-cualitativa presta un especial interés al papel de los cuerpos y la materialidad, lo que conlleva una especie de retiro de la metáfora de la mente y los procesos racionales. Lo que no deja de ser paradójico, cuando buena parte de los textos utilizan una serie de conceptos y un modo de escritura, que suponen un desafío de comprensión que se dirige hacia la racionalidad y no a la corporealidad. Por eso, parece contradictorio que dado el carácter altamente cognitivo, intelectual y abstracto del discurso de este movimiento se tome como referente los cuerpos y la materia que queda diluido en la propia urdimbre de los textos.

La pérdida de la representación. En algunos textos aparece un rechazo a la representación (MacLure, 2013; Martin y Kamberelis, 2013), como resultado de la necesidad de poner el énfasis en la materialidad. Lo que conlleva cuestionar lo que se considera una concepción estática y ordenada del mundo a través de representaciones mayormente lingüísticas del mismo. A lo que habría que unir el interés por realizar una

7 Esta cuestión surgió desde el público durante las Jornadas de sobre cómo aprenden los docentes: cartografías, en la mesa redonda What the new ontology brings to rethinking research and education. . dentro de las Jornadas ¿Cómo aprenden los docentes.? Enfoques cartográficos sobre el aprendizaje corpóreo, ecológico y nómada de los docentes (https://esbrina.eu/es/portfolio/enfoques-cartograficos-sobre-el-aprendizajecorporeo-ecologico-y-nomada-de-los-docentes/) Elizabeth de Freitas señaló que así había sido en una primera fase, pero que ahora cada vez más aparecen publicaciones que se articulan en torno a un caso. 
investigación productiva y no sólo reproductiva. Lo que choca con las formas que se utilizan para comunicar las propuestas y elaboraciones de las investigadoras post-cualitativas que utilizan formas representativas de carácter textual. Por nuestra parte, siempre hemos considerado, especialmente quienes transitamos entre imágenes, que la representación no es la realidad y que ha habido un exceso de representación textual y numérica de los fenómenos. Por eso tratamos de ampliar las formas representativas más allá de los textos, como hacemos en la investigación de la que dan cuenta varios artículos de este monográfico, pero nos resulta difícil pensar formas de compartir nuestras experiencias que no sean mediante representaciones.

La pérdida de la experiencia. Movidos por la influencia de autores como Deleuze, Guattari y Barad parece que la investigación social ha de transformarse en investigación filosófica. Que la invitación de Deleuze y Guattari a que la filosofía sea generadora y se articule en torno a conceptos, parece configurarse como una norma en la perspectiva post-cualitativa. En nuestra experiencia hemos considerado un terreno fructífero para abrir otros sentidos de la investigación favorecer un diálogo entre conceptualizaciones abstractas del mundo y la práctica de la investigación. En esta misma línea, compartimos el cómo se ha planteado el papel del "yo" en la investigación cualitativa (Hernández-Hernández y Sancho-Gil, 2018), especialmente en la de carácter autobiográfico convencional. Pero no podemos eludir que estamos implicados y somos afectados por las relaciones en las que entramos en la investigación.

La pérdida del sentido social. Una de las críticas a la investigación post-cualitativa es que parece dirigirse más a los académicos, pero que no tiene en cuenta -que elude- la función que la investigación desempeña en la sociedad, así como a los intereses que sirve. Esta crítica en el ámbito de la investigación post-cualitativa relacionada con la educación, pues parece eludir las relaciones históricas y políticas relacionadas con el poder social, y cómo estas afectan a la producción de conocimiento (Gerrard, Rudolph y Sriprakash, 2016) resulta pertinente considerarla.

A pesar de estos cuestionamientos, la perspectiva post-cualitativa conlleva la invitación a realizar una investigación que no separe ontología, epistemología, metodología y ética. Es decir, una investigación que preste atención a los entramados entre lo humano, lo no humano y lo material, Una propuesta que plantea asumir la investigación no como trayecto que define de antemano el camino a seguir respecto al fenóme- 
La perspectiva post-cualitativa en la investigación educativa: genealogía, movimientos, posibilidades y tensiones

Fernando Hernández-Hernández y Beatriz Revelles Benavente

no sobre el que se trata de ofrecer alguna luz, sino que se deja sorprender por lo que acontece en el fluir de la investigación.

Lo que lleva a poner un especial énfasis en la escritura como proceso de investigación (Richardson y St. Pierre, 2005). Una escritura que da cuenta del trayecto que se sigue como proceso y que sería la base de la investigación. Trayecto que no es lineal, que no sigue límites estrictos ni estructuras normativas; que se diversifica, que puede comenzar y bifurcarse en cualquier momento. Que asume que al moverse sin una ruta prefijada puede generar una sensación de incertidumbre y pérdida de un conocimiento histórico estable, fijo, repetitivo y pre-conceptualizado.

Situarse en este "devenir", lleva a prestar atención a lo diverso, lo material y lo emergente. Permite, además, comenzando en cualquier lugar, permanecer (por lo menos temporalmente) y no eludir pérdidas e incertidumbres. Lo que no significa inmovilismo, sino abrirse a la posibilidad de generar modos de pensar y prácticas de escritura que ofrezcan otras miradas sobre una realidad, que requiere ser considerada desde otros modos de pensar a indagar, y hacerlo a medida que se da cuenta de los tránsitos y desvíos que se recorren.

\section{Referencias}

American Education Research Association. (2006). Standards for reporting on empirical social science research in AERA publications. Educational Researcher, 35, 35-40.

American Education Research Association. (2009). Standards for reporting on humanities oriented research in AERA publications. Educational Researcher, 38, 481-486.

Ahmed, S. (2010). The promise of happiness. Durham, D.C: Duke University of Press

Atkinson, D. (2011). Art, Equality and Learning: Pedagogies Against the State. Rotterdam: Sage.

Atkinson, D. (2015). The adventure of pedagogy, learning and the not-known. Subjectivity, 8(1), 43-56.

Atkinson, D. (2018). Art, Disobedience, and Ethics. The Adventure of Pedagogy. London: Palgrave Macmillan.

Barad, K. (2003). Posthumanist Performativity: Toward an Understanding of How Matter Comes to Matter. Signs: Journal of Women in Culture and Society 28 (3), 801-31.

Barad, K. (2007). Meeting the Universe Halfway: Quantum Physics and the Entanglement of Matter and Meaning. Durham, NC: Duke University Press.

Barad, K. (2010). Quantum Entanglements and Hauntological Relations of Inheritance: Dis/ continuities, SpaceTime Enfoldings, and Justice-to-Come, Derrida Today. 3, 240 - 68.

Barad, K. (2014). Diffracting Diffraction: Cutting Together-Apart. Parallax, 20(3), 168-187, DOI 10.1080/13534645.2014.927623 
La perspectiva post-cualitativa en la investigación educativa: genealogía, movimientos, posibilidades y tensiones

Fernando Hernández-Hernández y Beatriz Revelles Benavente

Bargetz, B. (2014). Mapping Affect. Challenges of Un(timely) politics. In M. Angerer B. Bosel y M. Ott (eds). Timing of affect. Epistemologies, Aesthetics, Politics. (pp. 289 302). Zurich: Diaphanes..

Berlant, Laurent (2011). Cruel Optimism. Durham, NC: Duke University Press

Braidotti, R. (2015). Lo PosthumanoBarcelona: Gedisa.

Brizuela, B.M., Stewart, J.P., Carrillo, R.G. y Berger, J.G. (eds.) (2000). Acts of Inquiry in Qualitative Research. Cambridge, Mass.: Harvard Educational Revie.

Camps, V. (2011). El gobierno de las emociones. Barcelona : Herder

Casey, K . (1995-96). The new narrative research in education. Review of research in Education, 21, 211-253.

Clough, P. (a 2009). The new empiricism. Affect and Sociological method. European Journal of Social Theory, 12 (1), 43-61.

Clough, P. (2008). (De)Coding the Subject-in-Affect. Subjectivity, 23(1), 140-155. http:// dx.doi.org/10.1057/sub.2008.16

Coleman, R. (2013). A Sensory sociology of the future: Affect, hope and inventive methodologies. The Sociological Review. 65 (3), 525 - 543.

Coleman, R., Ringrose, J. (2013). Deleuze and Research Methodologies. Edinburgh: Edinburgh University press.

Colman, F. (2010).Affective Self: Feminist Thinking and Feminist Actions" Contemporary French and Francophone Studies 14 (5), 543 - 552

Committee on Scientific Principles for Education Research. (2002). R. J. Shavelson y L. Towne (Eds.), Scientific research in education. Washington, DC: National Research Council.

Connelly, M. y Clandinin, J. (1990). Stories of experience and narrative inquiry. Educational Researcher, 19 (4), 2-14.

Connelly, M. y Clandini, J. (2000). Narrative inquiry. San Francisco: Jossey-Bass.

Deleuze, G. (1968/1996). Spinoza y el problema de la expresión. Barcelona: Muchnick editores.

Cooper, K. y White, R. (2012). Qualitative Research in the Post-Modern Era. Dordrecht, Heidelberg, London, New York: Springer

Cherryholmes, C. (1988). Power and Criticism. Poststructural Investigation in Education. New York: Teachers College Press.

Creswell, J. W., Shope, R., Clark, V. L. P., y Green, D. O. (2006). How interpretive qualitative research extends mixed methods research. Research in the Schools, 13(1), 1-11.

Cvetkovich, A. (2012). Depression: A Public Feeling. Durham and London: Duke University Press.

Deleuze, G. (1981/2009). Spinoza: Filosofía práctica. Barcelona: Tusquets.

Deleuze, G. y Guattari, F. (2010). Mil Mesetas. Capitalismo y Esquizofrenia. Valencia: Pre-textos.

Deleuze, G. y Guattari, F. (1993). ¿Qué es la filosofía? Barcelona: Anagrama.

Denzin, N. K. (1997). Interpretative Ethnography. Thousand Oaks, CA: Sage.

Denzin, N. K. y Lincoln, Y. S. (eds). (2002). The Qualitative Inquiry Reader. Thousand Oaks, CA: Sage.

Denzin, N. K., y Lincoln, Y. S. (2005). Introduction: The discipline and practice of qualita- 
La perspectiva post-cualitativa en la investigación educativa: genealogía, movimientos, posibilidades y tensiones

Fernando Hernández-Hernández y Beatriz Revelles Benavente

tive research. In N. K. Denzin y Y. S. Lincoln (Eds.), The Sage handbook of qualitative research (pp. 1-32). Thousand Oaks, CA: Sage.Denzin, N.K (2010). The Qualitative Manifesto. A call to Arms. Walnut Creek, California: Left Coast Press

Dolphijn, R., van der Tuin, I. (2012). New Materialism: Interviews \& Cartographies. Open Humanity Press. Recuperado 30 mayo 2017 desde http://openhumanitiespress.org/ books/download/Dolphijn-van-der-Tuin_2013_NewMaterialism.pdf

Dolphijn, R. y van der Tuin, I. (2013). A thousand tiny intersections: Linguisticism, Feminism, Racism and Deleuzian becomings. In A. Saldanha y J. M. Adams (eds.) Deleuze and Race (pp. 129 - 43). Edinburgh: Edinburgh University Press,

Ellingson, L. y Ellis, C. (2008). Autoethnography as Constructionist Project. En J. A. Holstein \& J. F. Gubrium (eds.). Handbook of constructionist research (pp. 445-465). New York: The Guildford PressEnciso Domínguez, G. y Lara, A. (2014). Emociones y ciencias sociales en el s. XX: La precuela del giro afectivo. Athenea Digital, 14(1), 263288. http://dx.doi.org/10.5565/rev/athenead/v14n1.1094

Gerrard, J., Rudolph, S. y Sriprakash, A. (2016). The Politics of Post-Qualitative Inquiry: History and Power. Qualitative Inquiry, 23 (5), 384-394.

Greene, J. C. (2008). Is Mixed Methods Social Inquiry a Distinctive Methodology? Journal of Mixed Methods Research, 2, 7-22.

Greene, J. C. (2013) On rhizomes, lines of flight, mangles, and other assemblages, International Journal of Qualitative Studies in Education, 26 (6), 749-758, DOI: 10.1080/09518398.2013.788763

Greco, M., y Stenner, P.(2008). Emotions: a social science reader. London: Routledge.

Haraway, D. (1988). Situated Knowledges: The Science Question in Feminism and the Privilege of Partial Perspective. Feminist Studies, 14(3), 575-599.

Haraway, D. (1991). Simians, Cyborgs, and Women. The Reinvention of Nature. New York: Routledge

Haraway, D. (2008). When species meet. Minneapolis: University of Minessota Press.

Haraway, D. (2016). Staying with the trouble. Making kin in the Chthulucene. Durham, D.C: Duke University Press

Hernández, F. (2011). Pensar la relación pedagógica en la universidad desde el encuentro entre sujetos, deseos y saberes. En F. Hernández (ed). Pensar la relación pedagógica en la universidad desde el encuentro entre sujetos, deseos y saberes, (pp. 12-18) Barcelona: Repositorio digital de la Universitat de Barcelona.

Hernández-Hernández, F. (2015). Introducción al seminario Investigación post-cualitativa. Programa de doctorado Artes y Educación. Barcelona: Universidad de Barcelona (no publicado).

Hernández, F., y Rifà, M. (coords.) (2011). Investigación autobiográfica y cambio social. Barcelona: Octaedro.

Hernández-Hernández, F., y Sancho, J. M. (2015). A learning process within an education research group: an approach to learning qualitative research methods International Journal of Social Research Methodology, 18(6), 651-667. DOI: 10.1080/13645579.2015.1049468

Hernández-Hernández, F., y Sancho, J. M. (2018). Historias de vida y narrativas sobre la 
La perspectiva post-cualitativa en la investigación educativa: genealogía, movimientos, posibilidades y tensiones

Fernando Hernández-Hernández y Beatriz Revelles Benavente

subalternidad: Afrontar el desafío de lo inabordable de la relación con el Otro. Educar, 54(1), 15-29. DOI: https://doi.org/10.5565/rev/educar.913

Hickey-Moody (en prensa). Feminist Affect: The surprise that we find in the past. London: Routledge

Hickey-Moody, A. (2016). Femifesta for Posthuman Art Education: Visions and Becomings. In C.A. Taylor et al. (eds). Posthuman Research Practices in Education (pp. 258 - 267). New York: Palgrave MacMillan,

Higgins, M. (2017). Post-qualitative mo(ve)ments: Concluding remarks on methodological response-abilities and being wounded by thought. Reconceptualizing Educational Research Methodology, 8(3), 89-101.

Hinton, P., y Teusch, P. Teaching with Feminist Materialisms. Utrecht: Atgender

Jackson, A. Y., y Mazzei, L. A. (2012). Thinking with Theory in Qualitative Research. London, New York: Routledge.

Jonson, R. B. and A. J. Onwuegbuzie (2004). Mixed Methods Research: A Research

Paradigm Whose Time Has Come. Educational Researcher, 33(7), pp.14-26.

Just, E., Grahn, W. (2017) (eds) Theories of Affect in Generic skills education: Adventurous Encounters. Cambridge Scholars Publishing

Kincheloe, J. L., y Berry, K. (2004). Rigour and Complexity in Educational Research. Conceptualizing the bricolage. Nueva York: Open University Press.

Kohler-Riessman, C. (2008). Narrative methods for the human sciences. Thousand Oaks, CA: Sage.Koro-Ljungberg, M. (2015). Reconceptualizing qualitative research. Methodologies without Methodology. London: Sage.

Koro-Ljungberg, M., Yendol-Hoppey, D., Smith, J. J., y Hayes, S. B. (2009). (E)pistemological awareness, instantiation of methods, and uninformed methodological ambiguity in qualitative research projects. Educational Researcher, 38(9), 687-699.

Lara, A. \& Enciso Domínguez, G. (2013). El Giro Afectivo. Athenea Digital, 13(3), 101 119. http://dx.doi.org/ 10.5565/rev/athenead/v13n3.1060

Lara, A. \& Enciso Domínguez, G. (2014). Ciencia, Teoría Social y Cuerpo en el Giro Afectivo: Esferas de Articulación. Quaderns de Psicologia, 16(2), 7-25. http://dx.doi. org/10.5565/rev/qpsicologia.1172

Lather, P. (2007). Getting lost: Feminist efforts toward a double(d) science. Albany, NY: State University of New York Press.

Lather, P. (2012). The ruins of neo-liberalism and the construction of a new (scientific) subjectivity. Cultural Studies of Science Education, 7, 1021-1025.

Lather, P. (2013) Methodology-21: what do we do in the afterward? International Journal of Qualitative Studies in Education, 26:6, 634-645, DOI: 10.1080/09518398.2013.788753

Lather, P., St. Pierre, E. A. (2013). Introduction: Post-qualitative research. International Journal of Qualitative Studies in Education, 26(6), 629-633. DOI: 10.1080/09518398.2013.788752

Macbeth, D. (2001). On "reflexivity" in qualitative research: Two readings, and a third. Qualitative Inquiry, 7 (1), 35-68.MacLure, M. (2013). Researching without representation? Language and materiality in postqualitative methodology. International Journal of Qualitative Studies in Education, 26(6), 658-667. doi: DOI: 10.1080/09518398.2013.788755 
La perspectiva post-cualitativa en la investigación educativa: genealogía, movimientos, posibilidades y tensiones

Fernando Hernández-Hernández y Beatriz Revelles Benavente

MacLure, M. (2013). Researching without representation? Language and materiality in post-qualitative methodology, International Journal of Qualitative Studies in Education, 26 (6), 658-667, DOI: 10.1080/09518398.2013.788755

Adrian D. Martin, A.D., y Kamberelis, G. (2013). Mapping not tracing: qualitative educational research with political teeth, International Journal of Qualitative Studies in Education, 26 (6), 668-679, DOI: 10.1080/09518398.2013.788756

Mason, J. (2006). Mixing methods in a qualitatively driven way. Qualitative Research, 6(1), 9-25.

Massumi, B. (1995). The Autonomy of Affect. Cultural Critique, 31, pp. 83-109. http:// dx.doi.org/10.2307/1354446 Massumi, B. (2002). Parables for the Virtual: Movement, Affect, Sensation. Durham, NC: Duke University Press Books.

Meacham, J. (2017). The Naughty Nineties Set the Stage for Our Reality-TV Presidency, Time, September, 25, 21.

Miñana Blasco, C. y Rodríguez, J.G. (2003). La educación en el contexto neoliberal. En Restrepo Botero, D.I. (ed). La falacia neoliberal. Crítica y alternativas. (pp. 285-321). Bogotá: Universidad Nacional de Colombia.

National Science Foundation (NSF). (2004). Workshop on Scientific Foundations of Qualitative Research. Niccolini, A. (2016) Animate affects: censorship, reckless pedagogies, and beautiful feelings, Gender and Education, 28 (2), 230-249.

Onsès, J. (2018). Documentación visual en los fenómenos de aprendizaje con estudiantes de primaria. Una indagación rizomática difractiva desde las teorías 'post'. Barcelona: Universidad de Barcelona (Tesis doctoral no publicada)

Packwood, A. y Sikes, P. (1996). Adopting a postmodern approach to research, International Journal of Qualitative Studies in Education, 9 (3), 335-345, DOI: 10.1080/0951839960090308

Revelles-Benavente, B. (2017). Affecting feminist pedagogies: Performing critical thinking in between social networking sites and contemporary literature. In E. Just y W. Grahn (eds.) Theories of Affect in Generic skills education: Adventurous Encounters. Cambridge Scholars Publishing, $131-150$

Revelles-Benavente, B. y González, A. M. (2019). Género en la Educación: Pedagogía y Responsabilidad Feminista en Tiempos de Crisis Política Madrid: Ediciones Morata

Revelles-Benavente, B. (2015). Materializing feminist theory: the classroom as an act of resistance. In P. Hinton y P.Treusch (eds.) Teaching with Feminist Materialisms, (pp. 53-66) Utrecht: Atgender

Revelles-Benavente, B. (2018). Material knowledges. Intra-acting van der Tuin's new materialism with Barad's agential realism. Enrahonar. An International Journal of Theoretical and Practical Reason, 60, 75-91.

Richardson, L., y St. Pierre, E. A. (2005). Writing: A Method of Inquiry. In N. K. Denzin, e Y. S. Lincoln (Eds.), The Sage handbook of qualitative research (pp. 959-978). Thousand Oaks, CA. : Sage Publications Ltd.

Saldaña, J. (2009). The Coding Manual for Qualitative Researchers. Thousand Oaks: SAGE Publications Inc.

Sedgwick, E. y Frank, A. (1995). Shame in the cybernetic fold: Reading Silvan Tomkins. Critical Inquiry, 21(2), 496-522. Recuperado de http://www.jstor.org/stable/1343932 
La perspectiva post-cualitativa en la investigación educativa: genealogía, movimientos, posibilidades y tensiones

Fernando Hernández-Hernández y Beatriz Revelles Benavente

Scheurich, J.J. (2001). Research Method in the Postmodern. London, Philadelphia: Routledge y Falmer Press,

Schwandt, T.A. (1997). Qualitative Inquiry. A Dictionary of Terms. Thousand Oaks: SAGE Publications Inc.

Spinoza, B. (1667/1980). Ética demostrada según el orden geométrico. Madrid: Narcea.. St. Pierre, E. (2011). Post Qualitative Research: The Critique and the Coming After. En N. K. Denzin e Y. S. Lincoln (eds.) The Sage Handbook of Qualitative Research (4a ed. Revisada), (pp. 611-625). Thousand Oaks: SAGE Publications Inc.

St. Pierre, E.A. (2014). A Brief and Personal History of Post Qualitative Research Toward "Post Inquiry". Journal of Curriculum Theorizing. 30, (2). Se puede acceder en : http:// journal.jctonline.org/index.php/jct/article/viewFile/521/stpierre.pdf

St. Pierre, E. (2016). The Empirical and the New Empiricism. Cultural Studies Critical Methodologies, 16(2) $111-124$

Steier, F. (ed) (1991). Research and Reflexivity. Thousand Oaks: Sage.

Tashakkori, A. y Teddlie, C. (Eds.) (2003). Handbook of mixed methods in social and behavioral research Thousand Oaks, CA: Sage.

Taylor, C.A. y Hughes, Ch. (eds.) (2016) Posthuman Research Practices in Education. New York: Palgrave MacMillan.

Tomkins, S. (1963). Affect, Imagery, Consciousness / Vol. II: The Negative Affects. (pp.) New York: Springer Publishing.

Tomkins, S. (1991). Affect, Imagery, Consciousness: The Negative Affects: Anger and Fear. New York: Springer Publishing Company.

Van der Tuin, I. (2015). Generational Feminism: New Materialist Introduction to a Generative Approach. Maryland: Lexington Books

Villanueva, P. (2017). De qué hablamos cuando hablamos de Proyecto Artístico. Derivas desde los artistas, la institución y el arte contemporáneo. Barcelona: Universidad de Barcelona (Tesis doctoral no publicada).

Weyland, K. (2005). Review of rethinking social inquiry: Diverse tools, shared standards. In H. Brady y D. Collier (Eds.), Perspectives on politics (Vol. 3, pp. 392-393). Lanham, MD: Rowman and Littlefield. 
